Contributions to Game Theory and Management, XIV, 103-112

\title{
Opinion Control Problem with Average-Oriented Opinion Dynamics and Limited Observation Moments*
}

\author{
Jingjing Gao and Elena Parilina \\ St. Petersburg State University, \\ 7/9 Universitetskaya nab., Saint Petersburg 199034, Russia \\ E-mail: st081293@student.spbu.ru, e.parilina@spbu.ru
}

\begin{abstract}
In the paper, we propose a model of opinion dynamics in the presence of a center of influence. The center aims in distributing the opinion closer to the target one minimizing the costs. We consider the case when the center takes into account only some fixed number of observations from the opinion trajectory and taking into account the difference between the agent's opinion and the socially desired opinion in these periods. The dynamics of the state variable is given by a linear difference equation. The player's cost is a linear quadratic function with respect to the state variables and the player's strategy. The Euler equation method is used to find the center's optimal strategy. Numerical simulations of the theoretical results are given.
\end{abstract}

Keywords: opinion dynamics, social network, linear-quadratic games, Eulerequation approach.

\section{Introduction}

People exchange information in various ways to form their own opinions. The transfer of information allows people with different views to change their minds until the opinions merge. The diversity and complexity of opinion transmission makes the social network model extremely widely used, which has led to a great interest in its evolution.

A Markov chain is a model of stochastic process describing a sequence of possible events in which the probability of occurrence of each event depends only on the state attained in the previous time. The De Groot (DeGroot, Morris, 1974) model was the first to use Markov process theory to model the dynamics of opinions in social networks. The model assumes that network members with fixed weights may influence the opinions of the selected members. The next opinion of a member is a linear combination of all opinions of the current members. In the paper (Bure et al., 2017), a modified version of the De Groot model is presented. The authors propose a dynamic model of opinion formation with two principals or two centers of influence with the given influence matrix. They obtain the existence conditions of the consensus and examine several extreme cases. The competitive models of opinion dynamics are considered in (Sedakov and Zhen, 2019), where the dynamic game of opinion formation is linear-quadratic. The authors found equilibria that represent the degree of influence of different information structures. A cooperative version of this game is examined in (Rogov and Sedakov, 2020). A dynamic model of opinion formation with two agents and one or two centers of influence is proposed in (Mazalov and Parilina, 2020) and the optimal or equilibrium intense of influence is found. The Nash equilibrium found in the paper helps to reduce

\footnotetext{
${ }^{*}$ Jingjing Gao is the corresponding author. The work of the second author is supported by the Russian Science Foundation (grant no. 17-11-01079).

https://doi.org/10.21638/11701/spbu31.2021.09
} 
the cost of having social network members close to the target opinion. The paper (Mazalov and Parilina, 2019) proposes a model of opinion dynamics with a star structure of the society. The Nash equilibrium is found by studying the opinion dynamics of different centers in the opinion competition game.

In some social networks, the opinions of some influential people contribute to the formation and change of one's opinion. When these influencers want to reach a consensus among the members of the network, they need to use some strategies such as putting money, spreading baiting remarks, etc. When we need to observe the dynamics of opinions in social networks, there are certain social costs. So how can we reduce these costs?

We propose a model of opinion dynamics in the society consisting of the finite set of agents with a unique center of influence called a player. We assume that the player has a limited number $k$ of periods at which he can observe the society opinion and compare it with the desirable one. The player takes into account not only the squared difference between the agent's opinion and the target one, but also the quadratic costs on the opinion influence. The player aims to minimize the linear-quadratic functional with respect to an average-oriented opinion dynamics.

In our model, the state of the system is defined by the vector of opinions of social network members. The state variable depends on the previous state value and the intense of influence on the agents during the previous period. We apply the Euler equation approach to find the optimal solution of the optimization problem described above.

The rest of the paper is organized as follows. We describe the Euler equation method and formulate the optimization problem with a given mean-directed opinion dynamics in Section 2. In Section 3, we prove the main results for the certain observation moments case and find the optimal strategy of the player and agents' optimal opinion trajectories. We also provide the results of numerical simulations in Section 3, and briefly conclude in Section 4.

\section{Model}

\subsection{The Euler Equation Approach}

Let $X \subset \mathbb{R}^{n}$ and $U \subset \mathbb{R}^{m}$ be the state space and the control set, respectively. Given an initial state $x_{0} \in X$, the state of a system evaluates with respect to dynamics

$$
x(t+1)=f_{t}(x(t), u(t)), t=0,1, \ldots, T-1 .
$$

The optimal control problem is to find a control $u(t) \in U$ maximizing functional

$$
\sum_{t=0}^{T} \delta^{t} r_{t}(x(t), u(t))
$$

with respect to the state dynamics equations (1) and a given initial condition $x(0)=$ $x_{0}$, where $r_{t}(x(t), u(t))$ is a reward or cost function of a player.

We can reformulate this problem in terms of the state trajectory $x(t)$. Suppose that we can express $u(t)$ from equation (1) as a function of $x(t)$ and $x(t+1)$, say $u(t)=q(x(t), x(t+1))$. Therefore, we can rewrite functional (2) in the following form:

$$
\sum_{t=0}^{T} \delta^{t} g_{t}(x(t), x(t+1)),
$$


where $g_{t}(x(t), x(t+1))=r_{t}(x(t), q(x(t), x(t+1))), t=0,1, \ldots, T-1$. The Euler equation approach gives the necessary conditions (see e.g. (González-Sánchez and Hernández-Lerma, 2013; Dechert, 1978; González-Sánchez and Hernández-Lerma, 2014)) of the optimal trajectory $x^{*}(t)$ that are ${ }^{1}$

$$
\frac{\partial g_{t-1}\left(x^{*}(t-1), x^{*}(t)\right)}{\partial y}+\delta \frac{\partial g_{t}\left(x^{*}(t), x^{*}(t+1)\right)}{\partial x}=0, \quad t=1, \ldots, T-1 .
$$

where $x$ is the first variable in $g_{t}$ and $y$ is the second variable in $g_{t-1}$.

We can notice that the problem considered in the paper belongs to the class of linear-quadratic optimization problems. We will apply the Euler-equation method to find the player's optimal strategy in the dynamic problem with average-oriented opinion dynamics (see Mazalov and Parilina, 2020).

\subsection{Optimization Problem of Opinion Control}

We consider a society consisting of $n$ agents. Each agent $i$ has an opinion $x_{i} \in$ $\mathbb{R}, i=1, \ldots, n$. The agents communicate with each other and change the opinion over time, which is assumed to be discrete and finite. Let opinion of agent $i$ at time $t$ be $x_{i}(t) \in \mathbb{R}, t=0, \ldots, T$. We also assume that there exists a player who influences the opinion of one particular agent, say agent 1 , with intensity $u(t) \in \mathbb{R}$ at time $t=0,1, \ldots, T-1$. In period $T$ the player does not influence the agent's opinion. The opinion of agent 1 changes depending on his own opinion, the average opinion of the society and influence of the player. The opinion of any agent $i=2, \ldots, n$ changes over time depending on his own opinion and the average opinion of the society. The opinion dynamics is defined by the following equations:

$$
\begin{gathered}
x_{1}(t+1)=x_{1}(t)+a_{1}\left(\frac{\sum_{j=1}^{n} x_{j}(t)}{n}-x_{1}(t)\right)+u(t), \\
x_{i}(t+1)=x_{i}(t)+a_{i}\left(\frac{\sum_{j=1}^{n} x_{j}(t)}{n}-x_{i}(t)\right), i=2, \ldots, n,
\end{gathered}
$$

with initial condition

$$
x_{i}(0)=x_{i}^{0}, \quad i=1, \ldots, n .
$$

In equations (5) and (6), constants $a_{i} \in \mathbb{R}^{+}, i=1, \ldots, n$ show the beliefs of the agents to the average opinion of the society. The higher the average opinion and the personal opinion of an agent, the higher the opinion of the agent at next time period.

In the model proposed in (Mazalov and Parilina, 2020), the authors take into account all time periods to check if the opinion of the players is different from the player's target one. Therefore, the cost function in (Mazalov and Parilina, 2020) was defined in the form:

$$
J(u)=\sum_{t=0}^{T-1} \delta^{t}\left(\sum_{i=1}^{n}\left(x_{i}(t)-s\right)^{2}+c u^{2}(t)\right)+\delta^{T} \sum_{i=1}^{n}\left(x_{i}(T)-s\right)^{2},
$$

where $\delta \in(0,1]$ is a discount factor and $c>0$ is the player's costs per unit of influence intense (see Mazalov and Parilina, 2020). In the next sections we modify the

\footnotetext{
${ }^{1} \mathrm{We}$ assume that the conditions of Theorem 2.1 in (González-Sánchez and Hernández-Lerma, 2013) are satisfied.
} 
objective of the player and find the player's optimal strategy in a new formulation of the problem.

\section{Case of Some Observation Moments}

We assume that the player can monitor the opinion level in the society only at some time moments along the state trajectory. Let he use $k$ periods $0 \leq t_{1} \leq$ $t_{2} \leq \cdots \leq t_{k} \leq T-1$, where $k<T-1$, to observe the situation with the dynamics equations. We have two agents 1 and 2 with state variables $x_{1}(t)$ and $x_{2}(t)$ respectively:

$$
\begin{gathered}
x_{1}(t+1)=x_{1}(t)+\frac{a_{1}}{2}\left(x_{2}(t)-x_{1}(t)\right)+u(t), \\
x_{2}(t+1)=x_{2}(t)+\frac{a_{2}}{2}\left(x_{1}(t)-x_{2}(t)\right),
\end{gathered}
$$

with initial condition

$$
x_{1}(0)=x_{1}^{0}, x_{2}(0)=x_{2}^{0} .
$$

The functional for player 1 to minimize is

$$
\begin{aligned}
J(u)= & \sum_{t=0}^{T-1} \delta^{t}\left(c u^{2}(t)\right)+\sum_{j=1}^{k} \delta^{t_{j}}\left(\left(x_{1}\left(t_{j}\right)-s\right)^{2}+\left(x_{2}\left(t_{j}\right)-s\right)^{2}\right) \\
& +\delta^{T}\left(\left(x_{1}(T)-s\right)^{2}+\left(x_{2}(T)-s\right)^{2}\right) .
\end{aligned}
$$

The next theorem gives the necessary conditions of the player's optimal strategy found using the Euler equation approach.

Theorem 1. Let $\left\{u^{*}(t): t=0, \ldots, T-1\right\}$ be the optimal strategy minimizing functional (12) subject to initial conditions (11) and state dynamics equations (9) and (10), and $\left\{\left(x_{1}^{*}(t), x_{2}^{*}(t)\right): t=0, \ldots, T\right\}$ be the corresponding state trajectory, then the optimal strategy $u^{*}(t), t=0, \ldots, T-1$ is

$$
u^{*}(t)=z^{*}(t+1)-A z^{*}(t)
$$

and corresponding optimal state trajectory $\left(x_{1}^{*}(t), x_{2}^{*}(t)\right), t=1, \ldots, T$ satisfy the system of equations:

$$
\left\{\begin{array}{l}
A c \delta z(t+1)+B z(t-1)-C z(t) \\
\quad+\frac{A c}{\delta} z(t-2)=0, t=2, \ldots, T-1 \\
D z(t)-E z(t-1)-A \delta c z(t+1)+\frac{A c}{\delta} z(t-2) \\
\quad=\left(a_{2}-\delta\right)\left(x_{2}(t)-s\right)+x_{2}(t-1)-s, t=t_{j}, j=2, \cdots, k \\
c(z(t)-A z(t-1))+\delta\left(z(t)+x_{2}(t)-s\right)=0, t=T \\
-\left(\frac{c}{\delta}-A^{2} c\right) z(T-1)+\frac{A c}{\delta} z(T-2)+\left(A c-\frac{a_{2}}{2}\right) z(T) \\
\quad=a_{2}\left(x_{2}(T)-s\right) \\
x_{2}(t+1)=x_{2}(t)+\frac{a_{2}}{2} z(t), t=1, \ldots, T-1,
\end{array}\right.
$$

where $z^{*}(t)=x_{1}^{*}(t)-x_{2}^{*}(t), A=1-\frac{a_{1}+a_{2}}{2}, B=A c+\frac{c}{\delta}-A^{2} c, C=A^{2} \delta c+A c-c$, $D=c-A^{2} \delta c+\delta+A c-\frac{a_{2}}{2}$ and $E=A c-\frac{c}{\delta}+A^{2} c-1$.

Proof. We represent a new variable $z(t)$ as

$$
z(t)=x_{1}(t)-x_{2}(t), t=0, \ldots, T .
$$


From state equations $(9),(10)$ taking into account expression of $z(t)$, we obtain the new state equations:

$$
\begin{gathered}
z(t+1)=A z(t)+u(t), \\
x_{2}(t+1)=x_{2}(t)+\frac{a_{2}}{2} z(t),
\end{gathered}
$$

with initial condition

$$
z(0)=x_{1}^{0}-x_{2}^{0}, x_{2}(0)=x_{2}^{0}
$$

where $A=1-\frac{a_{1}+a_{2}}{2}$.

We find an expression of $u(t)$ from (14) and obtain

$$
u(t)=z(t+1)-A z(t),
$$

with

$$
u(0)=z(1)-A z(0) .
$$

Substitute these expressions into $\sum_{t=0}^{T} \delta^{t} g_{t}(x(t), x(t+1))$, we can rewrite the functional in the following form:

$$
\begin{aligned}
J\left(z, x_{2}\right)= & c(z(1)-A z(0))^{2}+\sum_{t=0}^{T-1} \delta^{t}\left[c(z(t+1)-A z(t))^{2}\right] \\
& +\sum_{j=1}^{k} \delta^{t_{j}}\left[\left(z\left(t_{j}\right)+x_{2}\left(t_{j}\right)-s\right)^{2}+\left(x_{2}\left(t_{j}\right)-s\right)^{2}\right] \\
& +\delta^{T}\left[\left(z(T)+x_{2}(T)-s\right)^{2}+\left(x_{2}(T)-s\right)^{2}\right] .
\end{aligned}
$$

To minimize $J\left(z, x_{2}\right)$ under condition given by equations (15) and (16), we form the Lagrange function

$$
L\left(z, x_{2}, k\right)=J\left(z, x_{2}\right)+\sum_{t=1}^{T-1} k_{t}\left(x_{2}(t+1)-x_{2}(t)-\frac{a_{2}}{2} z(t)\right) .
$$

The first-order conditions are $\frac{\partial L\left(z, x_{2}, k\right)}{\partial z(t)}=0, t=1, \ldots, T$ and $\frac{\partial L\left(z, x_{2}, k\right)}{\partial x_{2}(t)}=0, t=$ $1, \ldots, T$.

First, we find the derivatives and get

$$
\begin{gathered}
\frac{\partial J\left(z, x_{2}\right)}{\partial z(t)}=\delta^{t-1} 2 c(z(t)-A z(t-1))-\delta^{t} 2 A c(z(t+1)-A z(t)), \\
t=1, \ldots, T-1, t \neq t_{j}, \\
\frac{\partial J\left(z, x_{2}\right)}{\partial z(t)}=\delta^{t-1} 2 c(z(t)-A z(t-1))-\delta^{t} 2 A c(z(t+1)-A z(t)) \\
+\delta^{t} 2\left(z(t)+x_{2}(t)-s\right), t=t_{j}, j=1, \ldots, k, \\
\quad \frac{\partial J\left(z, x_{2}\right)}{\partial x_{2}(t)}=0, t=1, \ldots, T-1, t \neq t_{j}, \\
\frac{\partial J\left(z, x_{2}\right)}{\partial x_{2}(t)}=\delta^{t}\left[2\left(z(t)+x_{2}(t)-s\right)+2\left(x_{2}(t)-s\right)\right], t=t_{j}, j=1, \ldots, k, k
\end{gathered}
$$


Second, we write the systems of the first-order conditions that are

$$
\begin{gathered}
\left\{\begin{array}{c}
c(z(t)-A z(t-1))-A \delta c(z(t+1)-A z(t))=\frac{a_{2}}{4} k_{t} \delta^{-t} \\
t=1, \ldots, T-1, t \neq t_{j} \\
c(z(t)-A z(t-1))-A \delta c(z(t+1)-A z(t)) \\
\quad+\delta\left(z(t)+x_{2}(t)-s\right)=\frac{a_{2}}{4} k_{t} \delta^{-t}, t=t_{j}, j=1, \cdots, k, \\
c(z(t)-A z(t-1))+\delta\left(z(t)+x_{2}(t)-s\right)=0, t=T,
\end{array}\right. \\
\left\{\begin{array}{l}
k_{t-1}+k_{t}=0, t=1, \ldots, T-1, t \neq t_{j}, \\
\delta^{t}\left[2 z(t)+4\left(x_{2}(t)-s\right)\right]-k_{t}+k_{t-1}=0, t=t_{j}, j=2, \ldots, k, \\
\delta^{t}\left[2 z(t)+4\left(x_{2}(t)-s\right)\right]+k_{t-1}=0, t=T,
\end{array}\right.
\end{gathered}
$$

with initial conditions $z(0)=x_{1}^{0}-x_{2}^{0}, x_{2}(0)=x_{2}^{0}$.

Excluding $k_{t}$ from systems (17) and (18), finally, we obtain the system of equations

$$
\left\{\begin{array}{l}
A c \delta z(t+1)+B z(t-1)-C z(t)+\frac{A c}{\delta} z(t-2)=0, t=2, \ldots, T-1, \\
D z(t)-E z(t-1)-A \delta c z(t+1)+\frac{A c}{\delta} z(t-2) \\
\quad=\left(a_{2}-\delta\right)\left(x_{2}(t)-s\right)+x_{2}(t-1)-s, t=t_{j}, j=2, \ldots, k \\
c(z(t)-A z(t-1))+\delta\left(z(t)+x_{2}(t)-s\right)=0, t=T \\
-\left(\frac{c}{\delta}-A^{2} c\right) z(T-1)+\frac{A c}{\delta} z(T-2)+\left(A c-\frac{a_{2}}{2}\right) z(T)=a_{2}\left(x_{2}(T)-s\right)
\end{array}\right.
$$

where $B=A c+\frac{c}{\delta}-A^{2} c, C=A^{2} \delta c+A c-c, D=c-A^{2} \delta c+\delta+A c-\frac{a_{2}}{2}$, and $E=A c-\frac{c}{\delta}+A^{2} c-1$.

The theorem is proved.

Example 1. Let the society is represented by two agents, and $a_{1}=0.5, a_{2}=$ $0.4, \delta=1, c=0.2$. The initial opinions of the agents are $x_{1}(0)=0.8, x_{2}(0)=0.3$. For time horizon $T=10$, the player only observes the odd periods and his target opinion is $s=0.6$. Using Theorem 1 , the optimal state and control trajectories are presented in Table 1. The optimal value for functional (12) is 0.89 .

Table 1. Optimal state and control trajectories.

\begin{tabular}{c|c|c|c|c|c|c}
$t$ & $t=0$ & $t_{1}=1$ & $t_{2}=3$ & $t_{3}=5$ & $t_{4}=7$ & $t=9$ \\
\hline$x_{1}(t)$ & 0.8 & 0.9255 & 0.2154 & 0.1841 & 1.1001 & 0.8726 \\
$x_{2}(t)$ & 0.3 & 0.4 & 0.5515 & 0.5312 & 0.336 & 0.5823 \\
$z(t)$ & 0.5 & 0.5255 & -0.3361 & -0.3471 & 0.7641 & 0.2903 \\
$u(t)$ & 0.2505 & -0.056925 & 0.419455 & -0.437895 & 0.046845 & -0.153865
\end{tabular}

For the same parameters and horizon $T=10$, we introduce optimal state (for both agents 1 and 2) and strategy trajectories in Figures 1 and 2 respectively. 


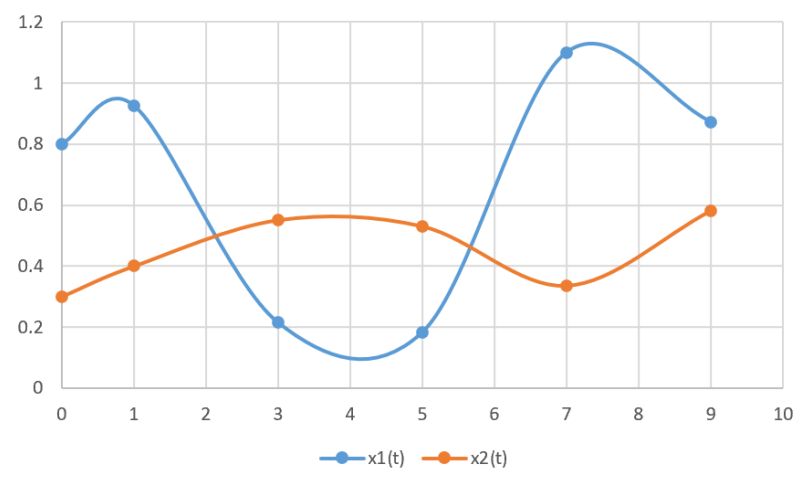

Fig. 1. Optimal state trajectories (blue $-x_{1}(t)$, red $-x_{2}(t)$ ).

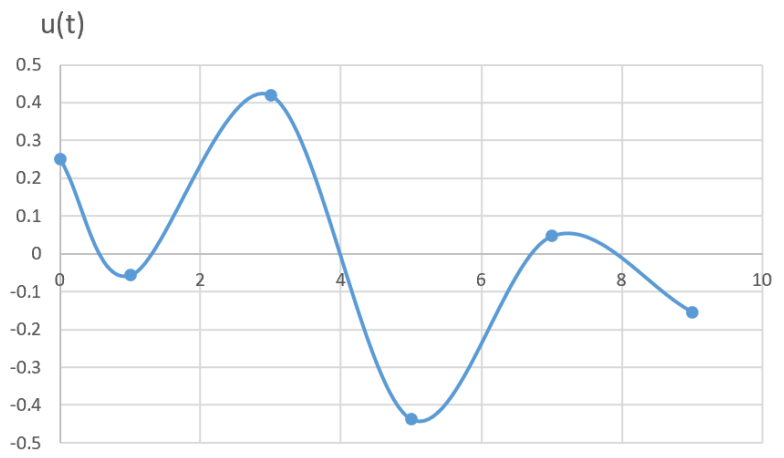

Fig. 2. Optimal strategy trajectory $u(t)$.

Example 2. Let $a_{1}=0.5, a_{2}=0.4, \delta=1, c=0.2$ and initial opinions be $x_{1}(0)=0.8, x_{2}(0)=0.3$. For time horizon $T=10$, the player observes the society opinion in time periods $2,3,5,6,9$. The target opinion is $s=0.6$. We find the optimal state and control trajectories presented in Table 2. The optimal value of functional (12) is 0.2 .

Table 2. Optimal state and control trajectories.

\begin{tabular}{c|c|c|c|c|c|c}
$t$ & $t=0$ & $t_{1}=2$ & $t_{2}=3$ & $t_{3}=5$ & $t_{4}=6$ & $t=9$ \\
\hline$x_{1}(t)$ & 0.8 & 0.5245 & 0.4509 & 0.4304 & 0.3862 & 0.6652 \\
$x_{2}(t)$ & 0.3 & 0.4429 & 0.4592 & 0.4639 & 0.4572 & 0.4662 \\
$z(t)$ & 0.5 & 0.0816 & -0.0083 & -0.0335 & -0.071 & 0.199 \\
$u(t)$ & -0.0605 & -0.05318 & 0.036165 & -0.052575 & 0.16565 & -0.01295
\end{tabular}

For the same parameters and time horizon $T=10$ we introduce optimal state and strategy trajectories in Figure 3 and 4 respectively. 


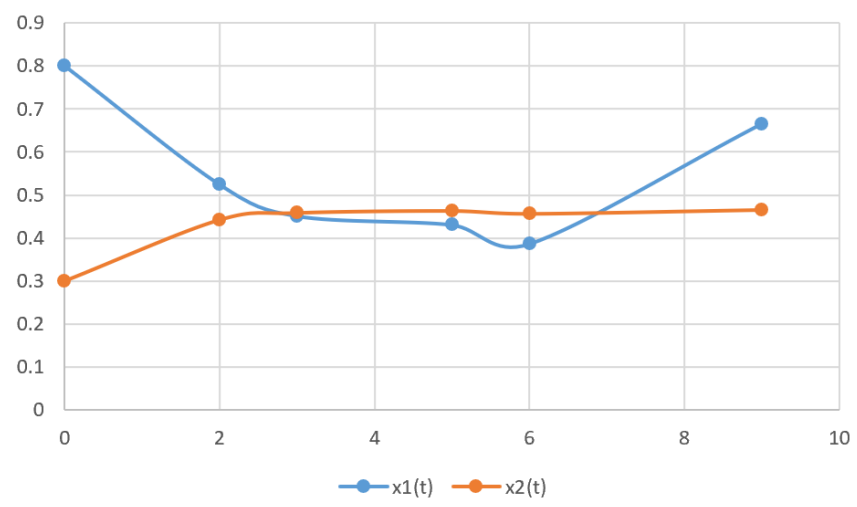

Fig. 3. Optimal state trajectories (blue $-x_{1}(t)$, red $-x_{2}(t)$ ).

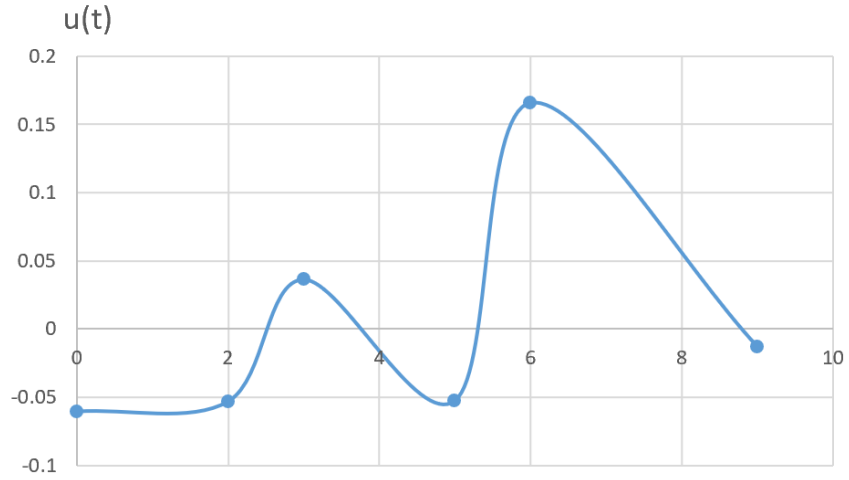

Fig. 4. Optimal strategy trajectory $u(t)$.

Example 3. Let $a_{1}=0.5, a_{2}=0.4, \delta=1, c=0.2$ and initial opinions be $x_{1}(0)=$ $0.8, x_{2}(0)=0.3$. For time horizon $T=10$, the player observes opinions on the society in periods 4,5 and 6,9 . His target opinion is $s=0.6$. The optimal trajectory and optimal control are presented in Table 3. The optimal value of functional (12) is 0.02 .

Table 3. Optimal state and control trajectories.

\begin{tabular}{c|c|c|c|c|c}
$t$ & $t=0$ & $t_{1}=4$ & $t_{2}=5$ & $t_{3}=6$ & $t_{4}=9$ \\
\hline$x_{1}(t)$ & 0.8 & 0.6613 & 0.6355 & 0.6243 & 0.5959 \\
$x_{2}(t)$ & 0.3 & 0.5746 & 0.5919 & 0.6006 & 0.6001 \\
$z(t)$ & 0.5 & 0.0867 & 0.0436 & 0.0237 & -0.0042 \\
$u(t)$ & -0.2269 & -0.004085 & -0.00028 & -0.031535 & 0.00251
\end{tabular}

For the same parameters and horizon $T=10$, we introduce state and strategy trajectories in Figures 5 and 6. 


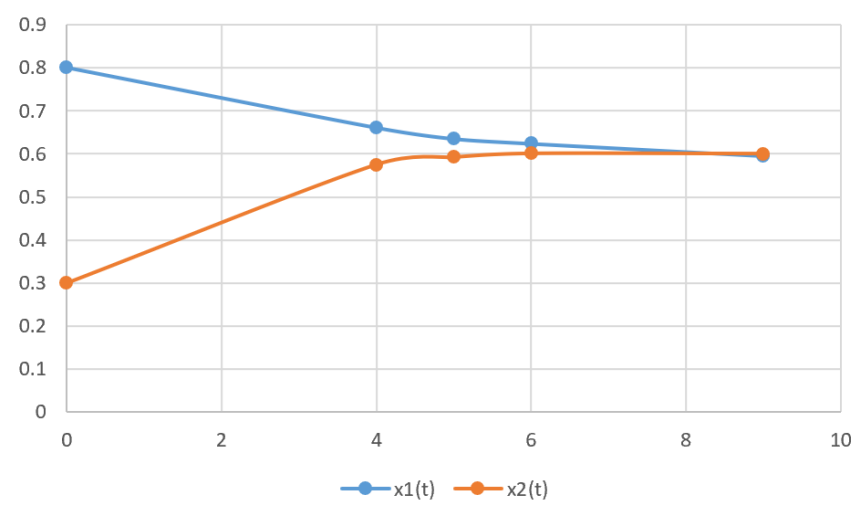

Fig. 5. Optimal state trajectories (blue $-x_{1}(t)$, red $-x_{2}(t)$ ).

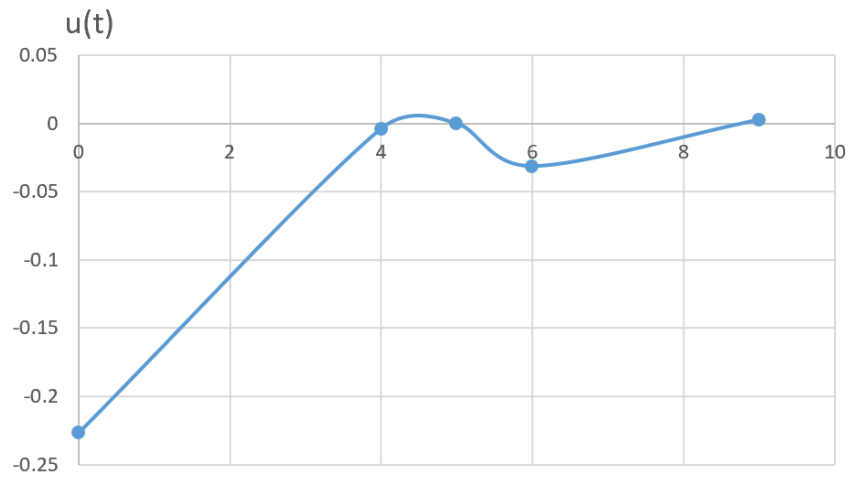

Fig. 6. Optimal strategy trajectory $u(t)$.

We compare the results of the numerical simulations from Examples 1-3. In Examples 1 and 2 the number of periods when the player observes the opinion of the society is the same, but in Example 1 the odd periods are chosen, while in Example 2 - the arbitrarily specified periods. The calculations show that the optimal value of the functional in Example 2 with prescribed moments of observation is less than in Example 1 with the odd moments of observations. The optimal control trajectories cannot be compared taking into account the moments of observations. The state trajectories seem to be more "stable" in Example 2. Example 3 demonstrates the state trajectory stabilization in the last periods of time interval. The value of the functional is significantly smaller in comparison with the ones in Examples 1 and 2. It can partially be explained by the smaller number of observations in comparison with Examples 1 and 2.

\section{Conclusions}

We propose a model of opinion dynamics in a society consisting of the finite number of agents with the presence of a center of influence called player. We consider the situation when the player cannot observe and compare the society opinion and 
his target one, but there is a given set of periods when the observations are made. The player observes the agents' opinions in arbitrary periods and takes them into account calculating the difference between the average agents' opinions and the socially desired one. We present the opinions of all members of the society as an arithmetic average of the opinions of the agents. We find the necessary conditions for the player's optimal strategy which is the intense of influence on a target agent. The Euler equation approach is used to define the optimal strategy. The numerical simulations verify theoretical results.

\section{References}

Bure, V. M., Parilina, E. M., and Sedakov, A. A. (2017). Consensus in a social network with two principals. Autom. Remote Control, 78(8), 1489-1499.

Dechert, D. (1978). Optimal control problems from second-order difference equations. J. Econ. Theory, 19(1), 50-63.

DeGroot, Morris H. (1974). Reaching a consensus. Journal of the American Statistical Association, 69(345), 118-121.

González-Sánchez, D. and Hernández-Lerma, O. (2013). Discrete-time stochastic control and dynamic potential games: the Euler-Equation approach. Chap. 2. Springer International Publishing: Cham, Switzerland.

González-Sánchez, D. and Hernández-Lerma, O. (2014). On the Euler equation approach to discrete-time nonstationary optimal control problems. J. Dyn. Games, 1(1), 57.

Mazalov, V. and Parilina, E. (2019). Game of competition for opinion with two centers of influence. In Mathematical Optimization Theory and Operations Research - 18th International Conference, MOTOR 2019, Proceedings, Russia, July 8-12, 2019 (Lecture Notes in Computer Science (Including Subseries Lecture Notes in Artificial Intelligence and Lecture Notes in Bioinformatics), 673-684. Springer: Cham, Switzerland.

Mazalov, V. and Parilina, E. (2020). The Euler-Equation Approach in Average-Oriented Opinion Dynamics. Mathematics, 8(3), 355.

Rogov, M. A. and Sedakov, A. A. (2020). Coordinated Influence on the Opinions of Social Network Members. Autom Remote Control 81, 528-547.

Sedakov, A. A. and Zhen, M. (2019). Opinion dynamics game in a social network with two influence nodes. Vestn. St. Petersburg Univ. Appl. Math. Comput. Sci. Control Process, 15(1), 118-125. 